\title{
A unified apparent porosity/permeability model of organic porous media: Coupling complex pore structure and multi- migration mechanism
}

\author{
Guanglong Sheng ${ }^{1}$, Yuliang $\mathrm{Su}^{2 \oplus *}$, Hui Zhao ${ }^{1}$, Jinghua Liu ${ }^{1}$ \\ ${ }^{1}$ School of Petroleum Engineering, Yangtze University, Wuhan 430100, P. R. China \\ ${ }^{2}$ School of Petroleum Engineering, China University of Petroleum (East China), Qingdao 266580, P. R. China
}

\section{Keywords:}

Shale gas reservoirs

organic matter

pore structure

multi-migration mechanism

apparent porosity

apparent permeability

Cited as:

Sheng, G., Su, Y., Zhao, H., Liu, J. A unified apparent porosity/permeability model of organic porous media: Coupling complex pore structure and multimigration mechanism. Advances in Geo-Energy Research, 2020, 4(2): 115-125, doi: 10.26804/ager.2020.02.01.

\begin{abstract}
:
Shale gas resources are widely distributed and abundant in China, which is an important field for strategic replacement and development of oil and gas resources. Shale gas reservoirs has adsorption gas, free gas. The structure of different scale media, such as organic pores, are difficult to describe. Therefore, flow behavior cannot be simulated by conventional method. In this paper, the micro-scale fluid migration in shale gas reservoirs was established in a single pore, which coupled surface diffusion, slip flow, and viscous flow. On this basis, the fractal scale relationship was applied to describe the distribution of pore radius, tortuosity, and surface roughness. Based on the comprehensive characterization of static structure characteristics of porous media, such as pore size distribution, pore shapes, tortuosity and surface roughness, and the dynamic pore size influenced by various stresses, the apparent porosity/permeability model of organic matter considering singlephase multi-migration mechanism was established. The gas migration in organic porous media was analyzed with the apparent porosity/permeability model. The results show that the small pores in organic matter are the main storage space of gas (more than $95 \%$ of the gas is stored in pores less than $10 \mathrm{~nm}$ ), and the large pores are gas flow channel. At the same time, the apparent porosity/permeability model combined with conventional Darcy equation can be used to describe the single-phase gas flow in shale gas reservoirs.
\end{abstract}

\section{Introduction}

Shale gas resources are widely distributed and rich in reserves, which will become an important area for China's oil and gas resources strategic replacement and development (Chen et al., 2011; Meng et al., 2019). Shale gas reservoirs have very different gas storage and pore structure from conventional gas reservoirs (Curtis et al., 2012; Wang et al., 2019). The shale pore structure is complex and multi-scale. Shale gas is generally stored as adsorbed phase and free phase (Wang and Reed, 2009; Wang et al., 2019). Shale gas mainly exists in the interior and surface of organic matter in adsorbed state, and it exists in the intergranular pores in adsorbed and free state. The nano-scale pores existing in shale cause a large internal surface area, and the adsorbed gas content may exceed $50 \%$ (Lu et al., 1995). The shale matrix is dense and the pore radius is very small. The pore radius of matrix is between $5-800 \mathrm{~nm}$, most of which are distributed around $100 \mathrm{~nm}$, and the radius of pore throat is generally 10-20 nm (Loucks et al., 2009).

The porous media of shale reservoirs has specific strong heterogeneity, and their pore size distribution, pore geometry, tortuosity, and surface roughness have a greater impact on fluid migration. Shale reservoirs have a wide range of pore size distribution. Loucks et al. (2009) studied Barnett shale in Mississippi, and found that organic nanopores are usually between 5 and $750 \mathrm{~nm}$. Wang et al. (2009) found the organic pore size range of Barnett shale is 5-1000 nm. Liu et al. (2016) found that organic matter in Qinshui Basin of China has pores larger than $1000 \mathrm{~nm}$. In fact, research showed that the pore size distribution of natural porous media, including organic matter in shale reservoirs, follow statistical fractal scales (Yang et al., 2014; Cai et al., 2018). To our best knowledge, there

\footnotetext{
${ }^{*}$ Corresponding author.

E-mail address: shengg12019@yangtzeu.edu.cn (G. Sheng); suyuliang@upc.edu.cn (Y. Su); zhaohui@yangtzeu.edu.cn (H. Zhao); liujh2019@yangtzeu.edu.cn (J. Liu). 2207-9963 (c) The Author(s) 2020. 
is few related studies comprehensively considered complex gas migration mechanism in nanopores and multi-scale radius distribution (Shi and Durucan, 2004).

Generally, pore geometry includes various possible shapes, including circular pores, elliptical pores, rectangular pores, slit pores, and square pores. SEM images from Eagle Ford shale samples showed that pores are mainly round, angular, and slitshaped pores (Afsharpoor and Javadpour, 2016; Song et al., 2019). In conventional oil and gas reservoirs, using circular pores approximation to represent actual pore type is reasonable because the pore surface-to-volume ratio in conventional reservoirs is small (Zhu et al., 2019). In shale reservoirs, pores have a large surface-to-volume ratio. Therefore, fluid flow in shales depends more on pore shape, especially when slip effect is significant (Wu et al., 2015; Xu et al., 2017; Li et al., 2018). The tortuous structure of porous media in shale reservoirs also has a great impact on fluid migration. For shale reservoirs, the tortuosity of micro-nano pores not only reflects the geometric characteristics of reservoirs (Mehmani et al., 2013; Singh and Javadpour, 2013), but also reflects fluid flow characteristics in porous media (Li et al., 2017), which needs to be comprehensively studied. In micro-nano porous media, the surface roughness changes pore radius distribution (crosssectional area), which affects free gas flow capacity (Wu et al., 2016). At the same times, surface roughness also increases surface area (cross-section perimeter). The surface roughness also making surface effect more significant (slip effect, surface diffusion, etc.), which need to be discussed in detail.

Scholars have done a lot of research on pore radius changing during depressurization of shale reservoirs. Compared with sandstone, organic matter has a weaker strength and is easily deformed, which is more affected by stress sensitivity (Dong et al., 2010). Related experiments showed that as effective stress increases, the porosity/permeability of porous media decreases significantly (Mokhtari et al., 2013). Considering that gas desorption on surface of shale organic matter is similar to that of coal, scholars have applied desorption shrinkage of coal to shale reservoirs (An et al., 2017). Saurabh and Harpalani (2018) proposed a fully coupled model that considers the effects of stress changes caused by adsorption on reservoir development. The study found that as pressure decreases, adsorbed gas is desorbed from micropores, causing rock matrix to shrink, which in turn enlarge pores.

The transport mechanism in organic media is complex (slip, adsorption and desorption, Knudsen diffusion, surface diffusion, etc.), and is affected by temperature, pressure, and surface properties (Xu et al., 2018; Chai et al., 2019; Du and Nojabaei, 2019; Wang and Sheng, 2019; Zuo et al., 2019). When gas flows in nanochannel, different slip boundary conditions can be described in different ranges of Knudsen numbers (Karniadakis et al., 2006; Song, 2012), which is defined as slip flow. Knudsen diffusion refers to the movement of free gas molecules caused by collision of molecules with pore surface. Based on related experiments, scholars have used a linear method to characterize microtubule gas diffusion under different pressure gradient, and have obtained Knudsen diffusion coefficients (Roy et al., 2003). Javadpour et al. (2007) proposed expression of microtubule Knudsen diffusion coef- ficients. Surface diffusion means that with pressure change in production process, the adsorbed gas diffuses due to chemical potential gradient, causing gas molecules to migrate along the streamline direction (Krishna and Wesselingh, 1997). It is considered that gas at the organic pore surface in shale reservoirs is single-layer adsorption, and the gas surface diffusion rate is much lower than gas adsorption desorption rate ( $\mathrm{Wu}$ et al., 2016).

When considering single-phase gas flow in shale reservoirs, scholars described multi-migration mechanism by establishing apparent permeability model, including Javadpours model based on pore size (Javadpour, 2009), Civans model based on Knudsen number (Civan, 2010) and DGM model based on diffusion coefficient (Freeman et al., 2011). Besides, the apparent porosity model was also proposed to couple gas storage with different states (Sheng et al., 2018, 2020). However, shale gas reservoirs have strong heterogeneity of porous media, large pore size distribution, diverse pore crosssectional shapes, complex tortuosity and surface roughness distribution. At the same time, pore radius is affected by stress changes during depressurization. There has no methods to comprehensively describe multi-migration mechanism, static structural characteristics, and dynamic pore radius changing in shale gas reservoirs.

\section{Multi-migration mechanism in a single pore}

Traditionally, transport mechanism in organic media including viscous flow, slip flow, adsorption and desorption, Knudsen diffusion, surface diffusion, and so on. Previous studies have shown that when considering a no-slip boundary, the velocity distribution of Poiseuille flow in a circle pore is (Mortensen et al., 2005):

$$
v(r)=\frac{R_{f}^{2}}{4 \mu_{g}}\left(1-r^{2}\right) \nabla p
$$

where $v$ is free gas velocity, $\mathrm{m} / \mathrm{s} ; R_{f}$ is pore radius for free gas, $\mathrm{m} ; \mu_{g}$ is gas viscosity, $\mathrm{Pa} \cdot \mathrm{s} ; \nabla p$ is the pressure gradient in flow direction, $\mathrm{Pa} / \mathrm{m}$.

The transport velocity of adsorption layer due to chemical potential gradient can be expressed as (Sheng, 2019):

$$
v_{a}=D_{a} C_{a} \frac{Z R T}{p^{2}} \nabla p
$$

where $v_{a}$ is transport velocity of adsorption layer, $\mathrm{m} / \mathrm{s} ; D_{a}$ is surface-diffusion coefficient, $\mathrm{m}^{2} / \mathrm{s} ; C_{a}$ is adsorbed gas concentration, $\mathrm{mol} / \mathrm{m}^{3} ; Z$ is compression factor for real gas, dimensionless; $R$ is universal gas constant, $8.314 \mathrm{~J} /(\mathrm{K} \cdot \mathrm{mol}) ; T$ is formation temperature, $\mathrm{K} ; p$ is pore pressure, $\mathrm{Pa}$.

The slip phenomenon was first introduced by Brown et al. (1946) in tube flow based on Maxwell theory, and the first-order boundary conditions based on Maxwell and aerodynamics were derived. There is a slip phenomenon in the flow of free gas near surface of micro- and nano-scale pores, and the slip phenomenon will make viscous flow easier. The slip coefficient is (Sheng, 2019): 


$$
F=1+\frac{2-f}{f} \frac{4 K n}{1-b K n}
$$

where $F$ is slippage coefficient, dimensionless; $f$ is tangential momentum accommodation coefficient (TMAC), dimensionless; $K n$ is Knudsen number in pores, dimensionless; $b$ is gas slip constant, dimensionless $(b=0$ represents fisrt-order slip boundary, $b=-1$ represents secondary-order slip boundary).

Knudsen diffusion is the movement of free gas molecules caused by collision of molecules with pore surface. The free gas flow caused by Knudsen diffusion is (Roy et al., 2003):

$$
N_{k}=\frac{1}{Z R T} \frac{2 R_{f}}{3} \sqrt{\frac{8 Z R T}{\pi M}} \nabla p
$$

where $N_{k}$ is gas flux of Knudsen diffusion per unit area, $\mathrm{mol} /\left(\mathrm{m}^{2} \cdot \mathrm{s}\right) ; M$ is molecular weight, $\mathrm{kg} / \mathrm{mol}$.

Without consider influence of adsorption layer, the ratio of additional gas flow caused by slip flow and Knudsen diffusion is:

$$
\frac{N_{s}}{N_{k}}=\frac{3 \pi}{16}\left(\frac{2}{f}-1\right)
$$

where $N_{k}$ is gas flux of slip flow per unit area, $\mathrm{mol} /\left(\mathrm{m}^{2} \cdot \mathrm{s}\right)$.

It can be seen that the ratio of flow rate caused by collision of gas molecules with wall from molecular dynamics theory (slip flow) and the flow rate obtained by collision between gas molecules and wall from experimental fitting (Knudsen diffusion) is constant. The value depends on TMAC. In particular, when $f$ is 0.74 , the calculation results are completely the same. Therefore, it can be considered that slip flow is same as that simulated by Knudsen diffusion. In actual simulation, It should be handled with slip boundary.

In conclusion, the multi-migration mechanism in nanopores of shale gas reservoirs can be expressed as:

$$
\begin{aligned}
q= & \frac{p}{Z R T} \frac{k_{f}}{\mu_{g}} A_{r} \frac{\Delta p}{l_{r}}+\frac{p}{Z R T} \frac{k_{a}}{\mu_{g}} A_{r} \frac{\Delta p}{l_{r}} \\
= & n_{p} A_{s e} D_{a} \frac{C_{a}}{p} \frac{\Delta p}{l_{b}}+\frac{n_{p} A_{f} p}{Z \mu_{g} R T}(1+\alpha K n) \frac{R_{f}^{2}}{8} \frac{\Delta p}{l_{p}} \\
& \times\left(1+\frac{2-f}{f} \frac{4 K n}{1-b K n}+D_{a} C_{a} \frac{16 M}{\pi \mu_{g}} K n^{2}\right)
\end{aligned}
$$

where $q$ is gas flux of cross-sectional area, $\mathrm{mol} /\left(\mathrm{m}^{2} \cdot \mathrm{s}\right) ; k_{f}$ is free gas permeability of porous medium, $\mathrm{m}^{2} ; A_{r}$ is crosssectional area, $\mathrm{m}^{2} ; l_{r}$ is rock length, $\mathrm{m} ; k_{a}$ is adsorbed gas permeability of porous medium, $\mathrm{m}^{2} ; n_{p}$ is pore number, dimensionless; $A_{f}$ is cross-sectional area for free gas, $\mathrm{m}^{2} ; \alpha$ is rarefaction coefficient, dimensionless; $l_{p}$ is pore length, $\mathrm{m}$; $A_{s e}$ is cross-sectional area for adsorbed gas, $\mathrm{m}^{2}$.

\section{Complex pore characterization}

\subsection{Multi-geometry shape coupling}

As previously analyzed, the pore shape distribution of porous media in shale reservoirs is complex, and three analytical approaches to describe fluid flow in porous medium filled with multi-geometry shape pores were proposed, including equivalent into one classic shape pore (Javadpour, 2009), corrected by geometry correction factor (Ren et al., 2016), and superimpose different shape pores (Singh et al., 2014). Sheng et al. (2020) used specific surface area and aspect ratio of height to width of noncircular nanopores to couple multi-geometry shape pores analytically. In their work, all of the pores were assumed to be classified into two types: elliptical pores (including circular pores and elliptical pores) and rectangular pores (including rectangular pores, slit pores, and square pores), as shown in Fig. 1.

The aspect ratio and specific surface area (SSA) of elliptical pores and rectangular pores has been defined in the work (Sheng et al., 2020). The multi-geometry pores were coupling by superimposing the pores with different range and frequency of SSA and aspect ratio.

\subsection{Pore radius distribution}

Studies have shown that the accumulated pore number with a pore radius greater than or equal to a characteristic length of a porous medium conforms to the fractal scale relationship (Majumdar and Bhushan, 1990; Yu and Cheng, 2002):

$$
N\left(L \geq R_{\text {int }}\right)=\left(\frac{R_{\max }}{R_{\text {int }}}\right)^{d_{r}}
$$

where $R_{\max }$ is the maximum pore radius, $\mathrm{m} ; d_{r}$ is the fractal dimension of the pore radius, dimensionless; $N$ is the number of pores; $R_{\text {int }}$ is pore radius with initial pore pressure, $\mathrm{m} ; R_{\max }$ is largest pore radius with initial pore pressure, $\mathrm{m}$.

Usually, we can obtain the maximum pore radius from experimental data, $\ln R_{\operatorname{maxt}}$, and get:

$$
\ln N\left(L \geq R_{\text {int }}\right)=d_{r} \ln \left(\frac{R_{\operatorname{maxt}}}{R_{\text {int }}}\right)+d_{r} \ln \left(\frac{R_{\max }}{R_{\operatorname{maxt}}}\right)
$$

where $R_{\operatorname{maxt}}$ is the maximum pore radius of cores from experimental data, $\mathrm{m}$.

Based on the above formula, the maximum pore radius of the porous medium can be obtained. The pore number distribution of different pore sizes is:

$$
n\left(R_{\text {int }}\right)=\frac{d N}{d R_{\text {int }}}=d_{r} R_{\max }^{d_{r}} R_{\text {int }}^{-\left(d_{r}+1\right)}
$$

where $n$ is the number of pores with radius of $R_{i n t}$.

Through a comparative study of pore size distribution data in the literature, as shown in Fig. 2. It can be seen that pore size distribution can be more accurately described based on fractal theory. At the same time, it can be seen that the fractal dimension of pore radius in the related literature are between 2.43-2.75.

\subsection{Fractal tortuosity}

Wheatcraft and Tyler (1988) first proposed the fractal relationship between flow path and pore length when fluid passes through random and complex porous media:

$$
l_{p}(\varepsilon)=\varepsilon^{1-d_{t}} l_{r}^{d_{t}}
$$




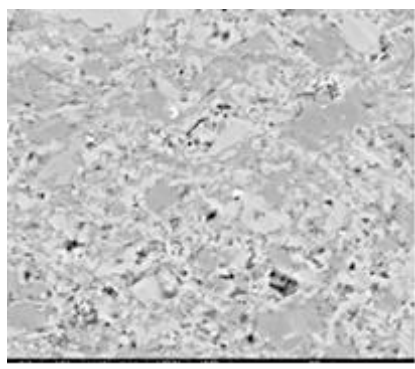

Actual multi-shape pore distribution
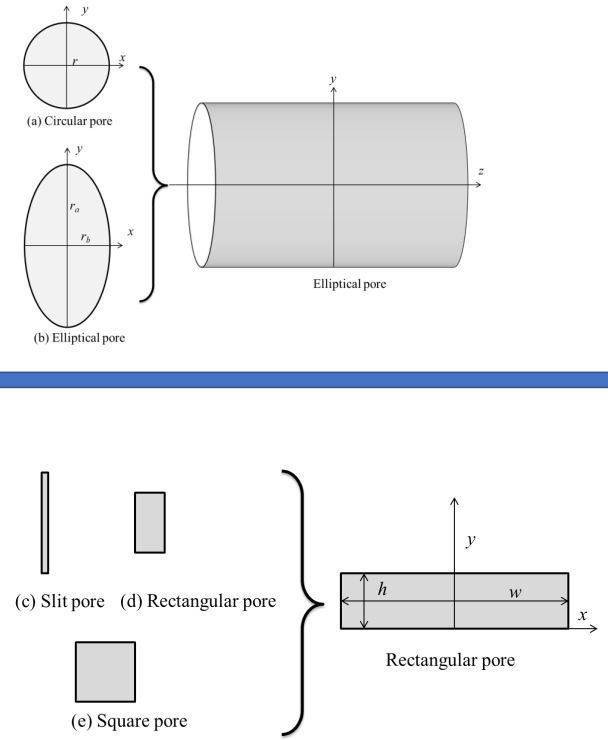

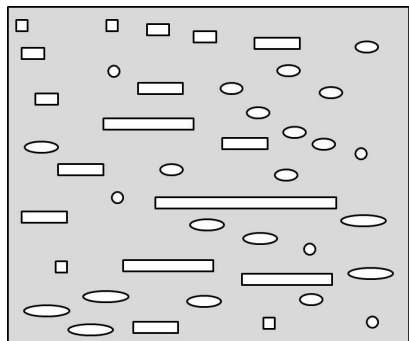

Idealized multi-shape pore distribution

Fig. 1. Multi-shape pores coupling in shale gas reservoirs (Sheng et al., 2020).

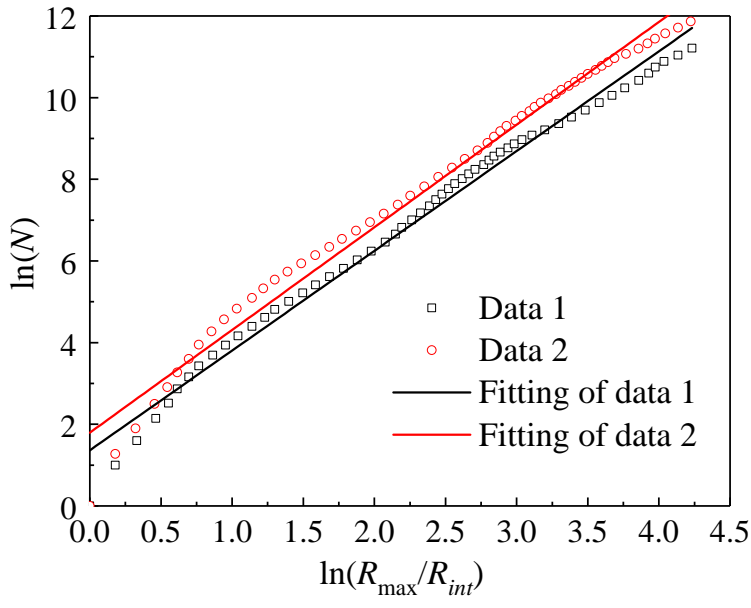

(a) Li and Zhang (2009)

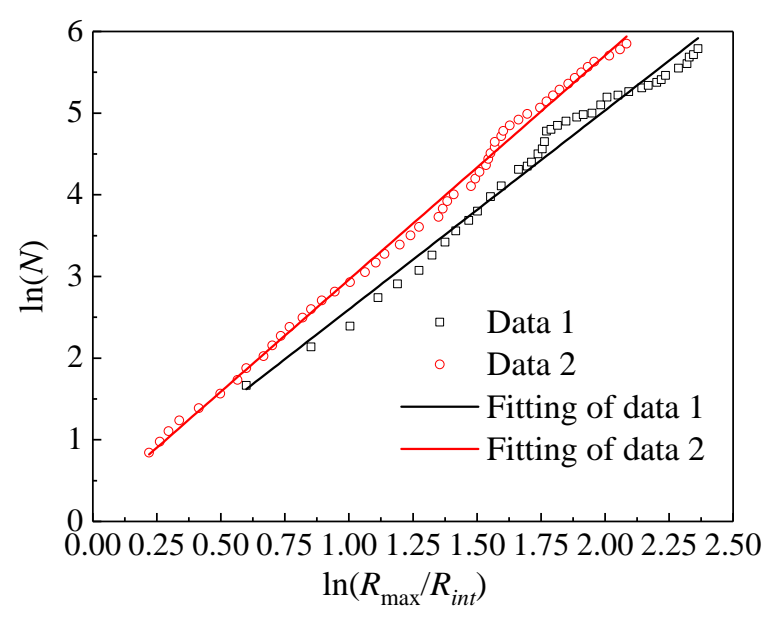

(b) Wang and Ju (2015)

Fig. 2. The fractal pore size distribution.

where $d_{t}$ is the fractal dimension of tortuosity, dimensionless; $\varepsilon$ is the scale for measurement in flow direction. When the measurement scale is equal to feature length, the tortuosity is 1 . When $\varepsilon$ approaches 0 , the tortuosity approaches infinity.

Yu and Cheng (2002) used pore radius as a measurement scale for single tubes, and proposed that pore length with different pore sizes can be expressed as a fractal scale relationship as:

$$
l_{p}\left(R_{i n t}\right)=R_{i n t}^{1-d_{t}} l_{r}^{d_{t}}
$$

The tortuosity of a single tube can be obtained as follows:

$$
\tau\left(R_{\text {int }}\right)=\tau\left(R_{\max }\right)\left(\frac{R_{\text {int }}}{R_{\max }}\right)^{1-d_{t}}
$$

where $\tau$ is tortuosity, dimensionless.

$\mathrm{Yu}$ and $\mathrm{Li}$ (2004) proposed a geometric model of flow paths tortuosity in porous media. The model assumes that in porous media, some particles cover each other without restriction, and some particles do not cover each other. The average tortuosity of porous media is:

$$
\tau_{\text {ave }}=\frac{1}{2}\left[1+\frac{1}{2} \sqrt{1-\phi}+\sqrt{1-\phi} \frac{\sqrt{\left(\frac{1}{\sqrt{1-\phi}}-1\right)^{2}+0.25}}{1-\sqrt{1-\phi}}\right]
$$

where $\tau_{\text {ave }}$ is average tortuosity, dimensionless; $\phi$ is porosity, dimensionless.

The fractal dimension of tortuosity in porous media can be calculated using the following equation:

$$
d_{t}=1+\frac{\ln \tau_{\text {ave }}}{\ln \left(\frac{R_{\max }}{R_{\text {ave }}}\right)}
$$

where $R_{\text {ave }}$ is average pore radius, $\mathrm{m}$. 


\subsection{Pore surface roughness}

The surface roughness change pore radius (cross-sectional area), which affects free gas flow capacity. Besides, surface roughness also increases surface area (cross-section perimeter), which makes surface effect more significant (slip flow, surface diffusion). Coppens and Dammers (2006) proposed the expression of actual area accessible to diffusion molecules:

$$
A_{s} \propto\left(\frac{d_{m}}{R_{\text {int }}}\right)^{2-d_{s}}
$$

where $A_{s}$ is actual area accessible by diffusion molecules, $\mathrm{m}^{2}$; $d_{s}$ represents fractal dimension of surface roughness. When $d_{s}$ equals 2 , it means a completely smooth plane. When $d_{s}$ equals 3 , it means that the surface completely fills the entire space. $d_{m}$ is diameter of gas molecules, $\mathrm{m}$.

It can be seen that when the difference between gas molecule and pore radius is small, the surface area is also small. That is, the surface roughness is not only the property of porous medium, but also the property of flowing molecules.

Taking into account the effect of surface roughness, the diffusion path of gas molecules on pore surface increases.
It reduces the effect of surface diffusion on the flow, which can be considered from perspective of tortuosity coefficient modification. We define the ratio of actual perimeter of pore section (the actual perimeter accessible to gas molecules) to equivalent perimeter (the perimeter calculated based on pore radius) as surface roughness tortuous, which is:

$$
\tau_{s}=\frac{l_{s}}{l_{b}}=\frac{A_{s}}{A_{b}}=\left(\frac{d_{m}}{R_{\text {int }}}\right)^{2-d_{s}}
$$

where $A_{b}$ is cross-sectional area calculated based on pore radius and pore cross-sectional shape, $\mathrm{m}^{2} ; \tau_{\mathrm{s}}$ is the roughness tortuosity of surface, dimensionless.

\section{Unified apparent porosity/permeability model}

\subsection{Apparent porosity}

The pore radius changing that comprehensively considers the effects of stress sensitivity, adsorption layer thinning, and desorption shrinkage can be obtained (Sheng et al., 2019). Based on this, the dynamic porosity of porous medium can be calculated as:

$$
\phi_{d c}=\frac{\int_{R_{\min }}^{R_{\max }} l_{p}\left(R_{\text {int }}\right) n_{p}\left(R_{\text {int }}\right) \sum_{i=1}^{\mathrm{N}} \varsigma_{i}\left(\pi E_{i} R_{d c}^{2}+4 R_{i} R_{d c}^{2}\right) d R_{\text {int }}}{\int_{R_{\min }}^{R_{\max }} l_{p}\left(R_{\text {int }}\right) n_{p}\left(R_{\text {int }}\right) \sum_{i=1}^{N}\left\{\frac{1-\phi_{\text {int }}}{\phi_{\text {int }}}\left[1-\varepsilon_{L} \frac{p_{L}\left(p_{\text {in }}-p\right)}{\left(p+p_{L}\right)\left(p_{\text {in }}+p_{L}\right)}\right] \varsigma_{i} R_{\text {int }}^{2}\left(\pi E_{i}+4 R_{i}\right)+\varsigma_{i} R_{d c}^{2}\left(\pi E_{i}+4 R_{i}\right)\right\} d R_{\text {int }}}
$$

where $\phi_{d c}$ is dynamic porosity of porous medium, dimensionless; $E_{i}$ is the ratio of elliptical pore number in each level to the total pore number, dimensionless; $R_{i}$ is the ratio of rectangular pore number in each level to the total pore number, dimensionless; $R_{d c}$ is dynamic pore radius, $\mathrm{m}$; $\varsigma_{i}$ is ratio of half-length of major axis to minor axis, dimensionless; $R_{\min }$ is the minimum pore radius, $\mathrm{m} ; \varepsilon_{L}$ is Langmuir strain, represents the maximum strain capacity of material, dimensionless; $\phi_{i n t}$ is porosity of initial pressure, dimensionless; $p_{L}$ is Langmuir's pressure, $\mathrm{Pa} ; p_{\text {in }}$ is initial pore pressure, $\mathrm{Pa}$.

Therefore, the porosity of adsorbed gas and free gas can be obtained as follows:

$$
\phi_{f}=\frac{\phi_{d c} \int_{R_{\min }}^{R_{\max }} l_{p}\left(R_{i n t}\right) n_{p}\left(R_{i n t}\right) \sum_{i=1}^{N}\left[\pi E_{i} \varsigma_{i} R_{d c}^{2}+4 R_{i} \varsigma_{i} R_{d c}^{2}-E_{i} \sqrt{\vartheta_{c e} \pi \varsigma_{i}} \frac{R_{d c} d_{m} p}{p_{L}+p}-4 R_{i}\left(1+\varsigma_{i}\right) \frac{R_{d c} d_{m} p}{p_{L}+p}\right] d R_{i n t}}{\int_{R_{\min }}^{R_{\max }} l_{p}\left(R_{\text {int }}\right) n_{p}\left(R_{\text {int }}\right) \sum_{i=1}^{N} \varsigma_{i}\left(\pi E_{i} R_{d c}^{2}+4 R_{i} R_{d c}^{2}\right) d R_{\text {int }}}
$$

where $\phi_{f}$ is free gas porosity of porous medium, dimension- less; $\vartheta_{c e}$ is SSA of elliptical pores, dimensionless.

$$
\phi_{a}=\frac{\phi_{d c} \int_{R_{\min }}^{R_{\max }} l_{p}\left(R_{\text {int }}\right) n_{p}\left(R_{\text {int }}\right) \sum_{i=1}^{N}\left[E_{i} \sqrt{\vartheta_{c e} \pi \varsigma_{i}} \frac{R_{d c} d_{m} p}{p_{L}+p}+4 R_{i}\left(1+\varsigma_{i}\right) \frac{R_{d c} d_{m p} p}{p_{L}+p}\right] d R_{\text {int }}}{\int_{R_{\min }}^{R_{\max }} l_{p}\left(R_{\text {int }}\right) n_{p}\left(R_{\text {int }}\right) \sum_{i=1}^{N} \varsigma_{i}\left(\pi E_{i} R_{d c}^{2}+4 R_{i} R_{d c}^{2}\right) d R_{\text {int }}}
$$

where $\phi_{a}$ is adsorbed gas porosity of porous medium, dimensionless.

Then the apparent porosity of porous media is:

$$
\phi_{a p p}=\phi_{f}+C_{a} \frac{Z R T}{p} \phi_{a}
$$

where $\phi_{a p p}$ is apparent porosity of porous medium, dimensionless.

\subsection{Apparent permeability}

Considering multi-scale pore distribution, the free gas and adsorbed gas permeability of different pore sizes and aspect ratio are:

$$
k_{f e}\left(R_{i n t}, \varsigma_{i}\right)=\psi_{f e m}\left(R_{i n t}, \varsigma_{i}\right) F_{e}\left(R_{i n t}, \varsigma_{i}\right)(1+\alpha K n) \frac{\varsigma_{i}^{2} \lambda_{d c}^{2}}{4\left(\varsigma_{i}^{2}+1\right)}
$$

$$
k_{f r}\left(R_{i n t}, \varsigma_{i}\right)=\psi_{f r m}\left(R_{i n t}, \varsigma_{i}\right) F_{r}\left(R_{i n t}, \varsigma_{i}\right)(1+\alpha K n) \frac{\lambda_{d c}^{2}}{3}
$$

$$
k_{a r}\left(R_{\text {int }}, \varsigma_{i}\right)=\psi_{a r m}\left(R_{i n t}, \varsigma_{i}\right) D_{a} C_{a} \frac{\mu_{g} Z R T}{p^{2}}
$$




$$
k_{a e}\left(R_{\text {int }}, \varsigma_{i}\right)=\psi_{a e m}\left(R_{\text {int }}, \varsigma_{i}\right) D_{a} C_{a} \frac{\mu_{g} Z R T}{p^{2}}
$$

where $k_{f r}$ is free gas permeability of porous medium filled with rectangular pores, $\mathrm{m}^{2} ; k_{a r}$ is adsorbed gas permeability of porous medium filled with rectangular pores, $\mathrm{m}^{2} ; k_{f e}$ is free gas permeability of porous medium filled with elliptical pores, $\mathrm{m}^{2} ; k_{a e}$ is adsorbed gas permeability of porous medium filled with elliptical pores, $\mathrm{m}^{2} ; F_{e}$ is slippage coefficient of elliptical pores, dimensionless; $F_{r}$ is slippage coefficient of rectangular pores, dimensionless.

The free gas and adsorption gas permeability correction coefficients of elliptic and rectangular pore are listed in Appendix A.

Considering fractal pore radius distribution, the apparent permeability of porous media is:

$$
\begin{aligned}
k_{a p p}= & \int_{R_{\min }}^{R_{\max }} \sum_{i=1}^{N}\left[k_{f e}\left(R_{i n t}, \varsigma_{i}\right)+k_{f r}\left(R_{\text {int }}, \varsigma_{i}\right)\right. \\
& \left.+k_{a e}\left(R_{\text {int }}, \varsigma_{i}\right)+k_{a r}\left(R_{\text {int }}, \varsigma_{i}\right)\right] d R_{\text {int }}
\end{aligned}
$$

where $k_{\text {app }}$ is apparent permeability, $\mathrm{m}^{2}$.

\section{Results and Discussion}

Fig. 3 shows the fractal tortuosity and average tortuosity calculated under different fractal dimensions of tortuosity. It can be seen that the fractal tortuosity gradually decreases with the increase of pore radius. When pore radius is small, the average tortuosity is smaller than the fractal tortuosity, and when the pore radius is large, the average tortuosity is greater than the fractal tortuosity. The corresponding apparent permeability distribution is shown in Fig. 4. It can be seen that when pore radius is less than $2 \mathrm{~nm}$, the apparent permeability is larger when the fractal tortuosity is not considered, and the larger the fractal dimension is, the larger the difference will be. The largest deviation ratio is about $40 \%$. With the increase of pore radius, the apparent permeability without considering fractal tortuosity variation characteristics is small, and the deviation increases with the increase of pore radius and fractal

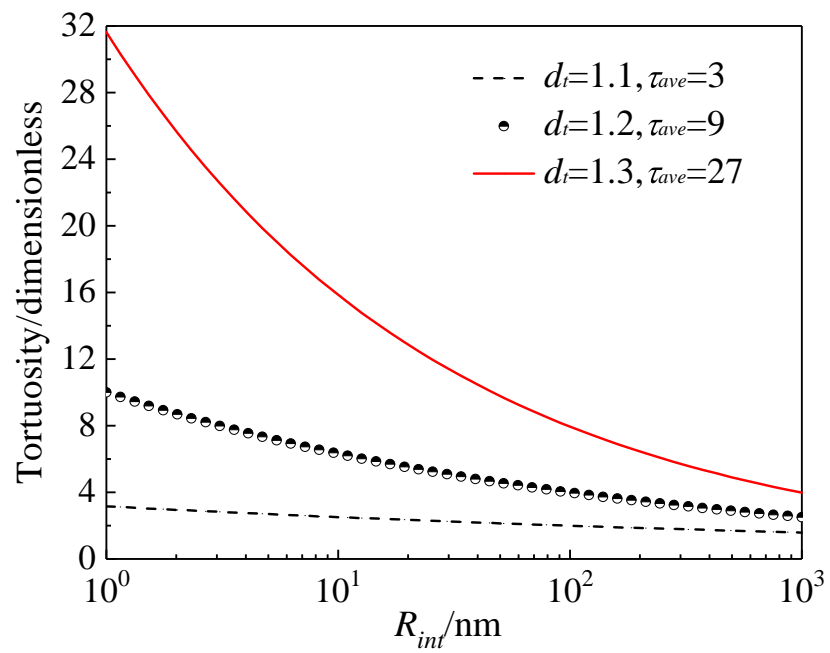

Fig. 3. Relationship of fractal tortuosity and average tortuosity with different fractal dimensions.

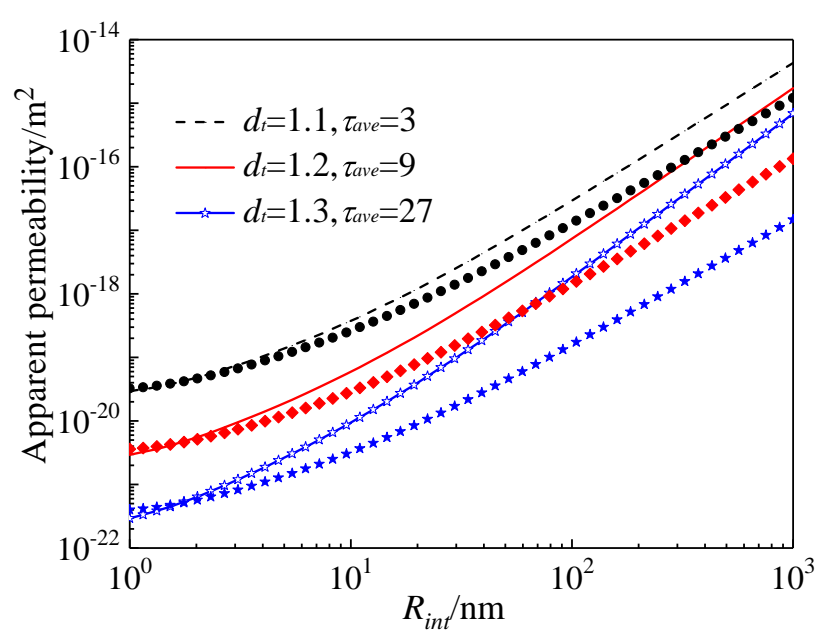

(a) Apparent permeability

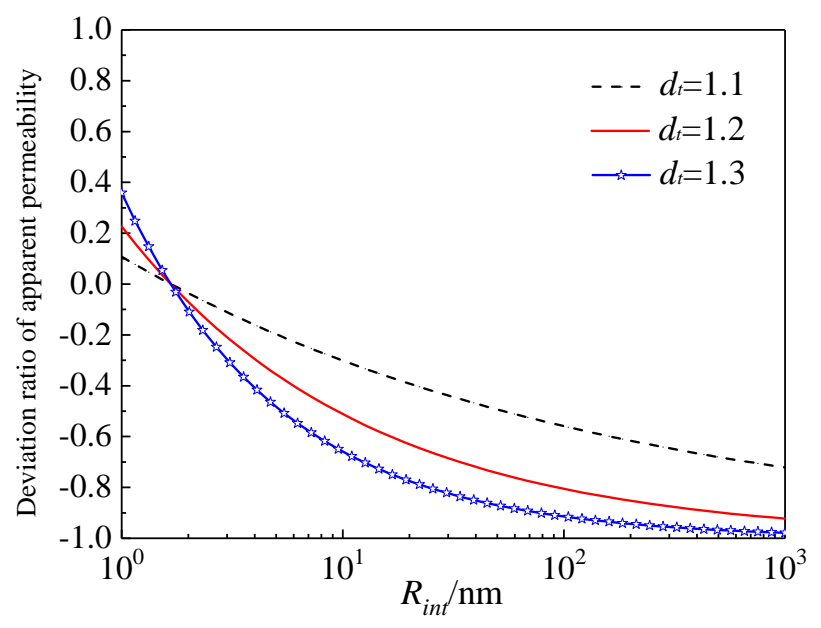

(b) Deviation ratio of apparent permeability

Fig. 4. Influence of fractal tortuosity on apparent permeability with different pore radius.

dimension. The maximum deviation ratio is $-98 \%$.

The surface roughness mainly changes apparent permeability by affecting adsorption gas migration distance and free gas migration cross-sectional area, which also has no effect on apparent porosity. Analyze the effect of surface roughness on apparent permeability, as shown in Fig. 5. It can be seen from the figure that the surface roughness mainly affects small pores. When pore radius is less than $100 \mathrm{~nm}$, the larger the surface roughness is (the larger the fractal dimension), the smaller the apparent permeability will be. The main reason is that when the pore radius is small, the proportion of adsorbed gas permeability in the apparent permeability is large, and the cross-sectional area of free gas is greatly affected by surface roughness. As pore radius increases, the effect of surface roughness on apparent permeability first increases and then decreases, as shown in Fig. 5(b). When the pore radius is between $3-5 \mathrm{~nm}$, the apparent permeability is most affected by surface roughness.

Assume that there is only one pore with the largest pore radius. It can be seen that the pore number increases exponen- 


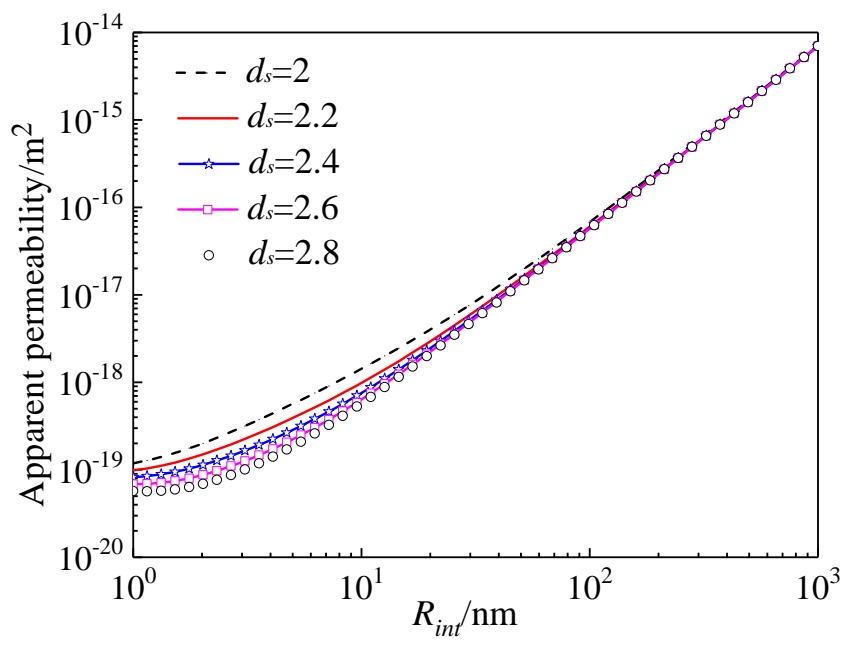

(a) Apparent permeability

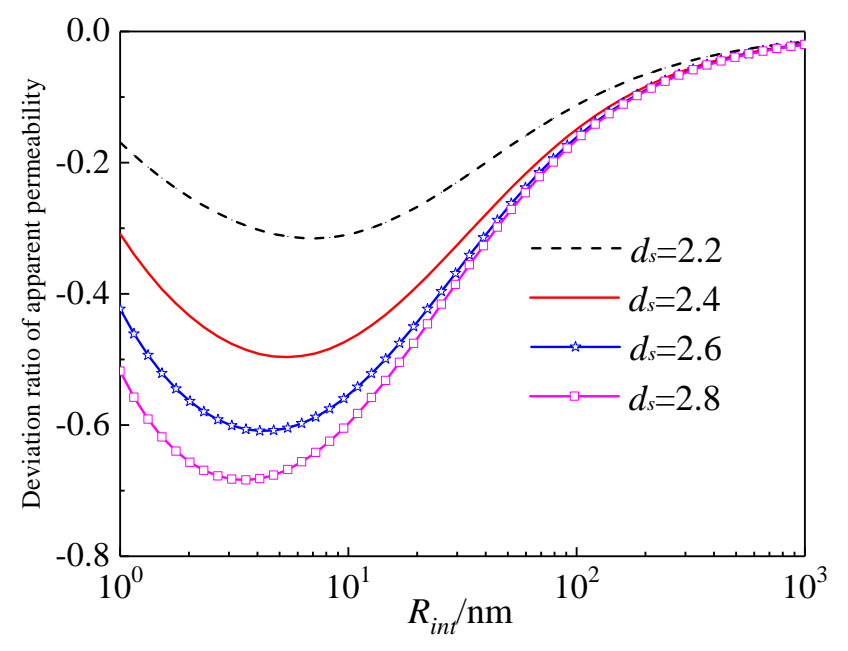

(b) Deviation ratio of apparent permeability

Fig. 5. Influence of surface roughness on apparent permeability with different pore radius.

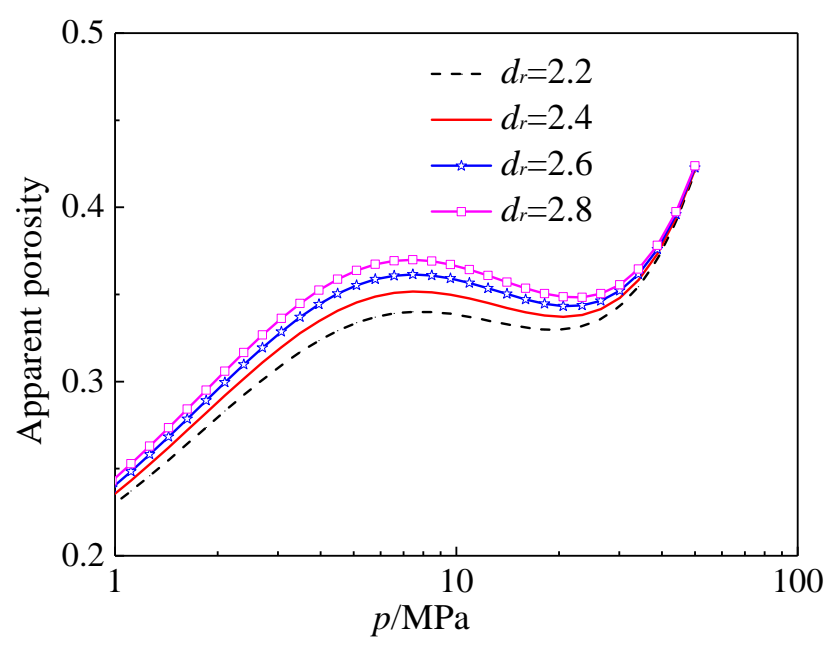

(a) Apparent porosity

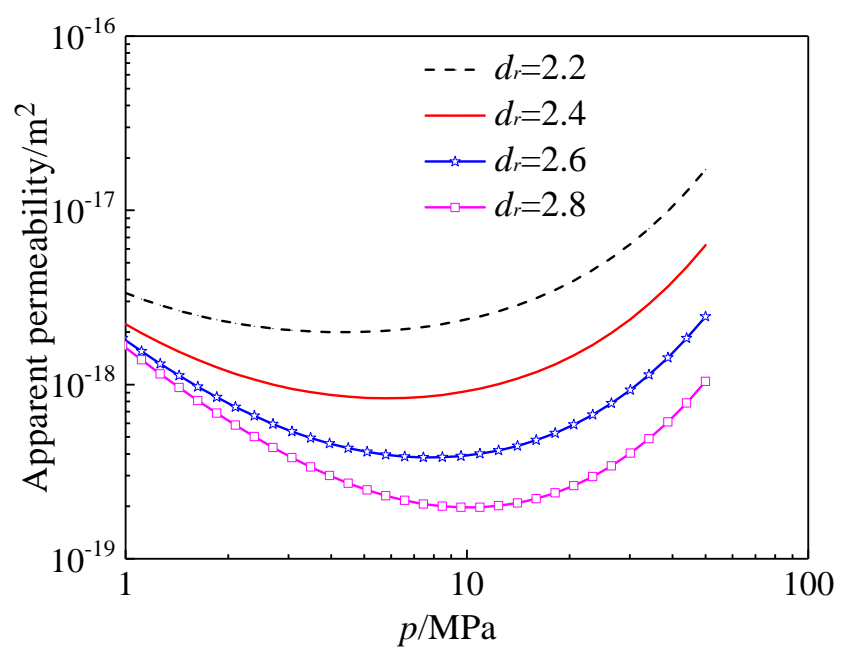

(b) Apparent permeability

Fig. 6. Influence of fractal pore distribution on apparent permeability with the same porosity.

tially as pore radius decrease. At the same porosity, the smaller the fractal dimension is, the larger the proportion of small pores will be. Fig. 6 shows the apparent porosity/permeability of porous medium coupling considering multi-scale pore. It can be seen that the apparent porosity first decreases, then increases, and finally decreases as pressure decreases. The main reason is that when pore pressure is greater than $20 \mathrm{MPa}$, the stress sensitivity plays a dominant role in pore changing. When pore pressure is in the range of 4-20 MPa, the desorption shrinkage dominates pore changing. When pore pressure continues to decrease, the amount of adsorbed gas on pore surface decreases, and the amount of desorption gas decreases accordingly. At this time, stress sensitivity plays a leading role. It can be also seen that pore radius distribution has a significant effect on apparent porosity when pore pressure between 1 and $20 \mathrm{MPa}$. The larger the fractal dimension is, the greater the effect of desorption shrinkage will be, and the larger the apparent porosity will be. Analyzing the effect of fractal dimension on apparent permeability, it can be seen that the fractal dimension has a greater effect on apparent permeability. The larger the fractal dimension is, the more the small pore will be, and the smaller the apparent permeability will be.

Under economic pore pressure (5 MPa), analyze the contribution of different radius pores to apparent porosity/permeability, as shown in Fig. 7. It can be seen that the smaller the pore radius is, the larger the contribution to apparent porosity will be. The small pore has a great effect on gas storage. This is because the smaller the pore radius is, the larger the surface-to-volume ratio will be, and the more the adsorbed gas will be. More than $95 \%$ of gas is stored in the pores below $10 \mathrm{~nm}$, and the larger the fractal dimension is, the higher the amount of gas in small pores will be, the higher the storage capacity will be. With the increase of pore radius, 


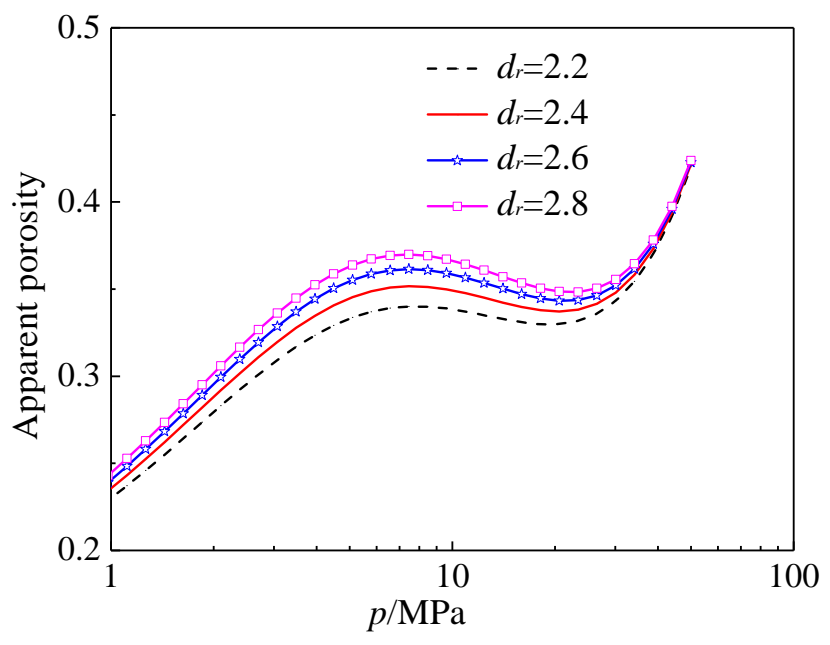

(a) Apparent porosity

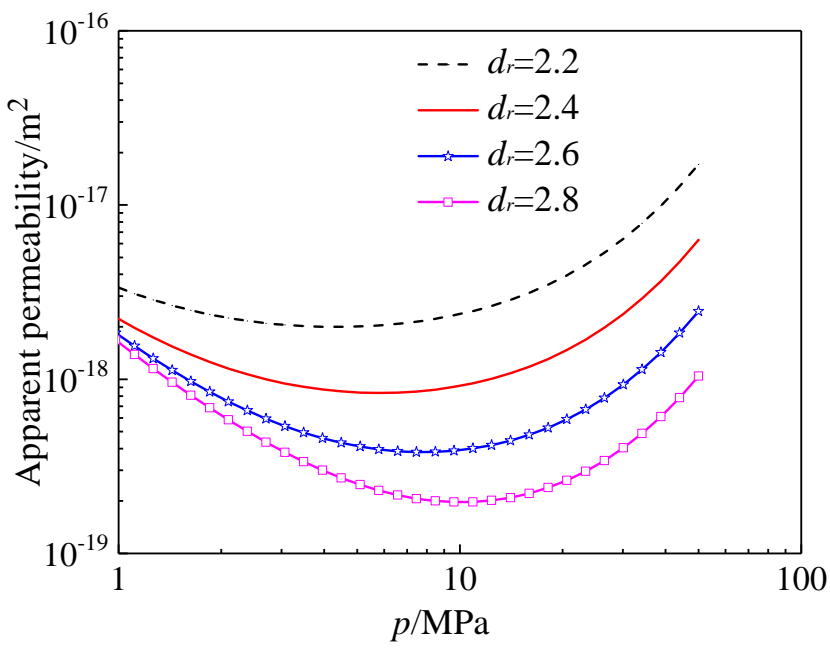

(b) Apparent permeability

Fig. 7. Contribution of different pore radius to apparent porosity/permeability of multiscale pores.

the contribution of different pores to apparent permeability decreases first and then increases, and its distribution is greatly affected by fractal dimension. For the same porosity, the larger the fractal dimension is, the higher the proportion of small pore will be, and the greater the contribution of small pores to apparent permeability will be. For the pores smaller than $100 \mathrm{~nm}$, the sum of their permeability contributions ranges from $14.5 \%$ to $61.5 \%$. Therefore, small pores in organic matter are gas storage media, and large pores are gas flow channels, similar to the relationship between matrix and fractures in conventional reservoirs.

\section{Conclusions}

In this paper, mathematical methods are used to quantitatively characterize static structure of porous media and dynamic pore radius. Combined with multiple transport mechanism in nanopores, the governing equations of gas migration in shale gas reservoirs and the apparent porosity/permeability model of porous media are obtained. The following conclusions were reached:

1) The tortuosity of different radius pore was described by fractal theory, and the results showed that fractal tortuosity gradually decreases with the increase of pore rdius. The fractal tortuosity has a great influence on transport capacity.

2) The influence of surface roughness on gas transport capacity was analyzed. Results showed that the surface roughness is not only the property of porous medium, but also the property of flowing molecules. The surface roughness mainly affects small pores.

3) The gas migration in organic porous media was analyzed. The results showed that the small pores in organic matter are the main storage space of gas (more than $95 \%$ of the gas is stored in pores less than $10 \mathrm{~nm}$ ), and the large pores are gas flow channel.

\section{Acknowledgement}

This study was supported by the National Natural Science Foundation of China (51922007).

\section{Conflict of interest}

The authors declare no competing interest.

Open Access This article, published at Ausasia Science and Technology Press on behalf of the Division of Porous Flow, Hubei Province Society of Rock Mechanics and Engineering, is distributed under the terms and conditions of the Creative Commons Attribution (CC BY-NC-ND) license, which permits unrestricted use, distribution, and reproduction in any medium, provided the original work is properly cited.

\section{References}

Afsharpoor, A., Javadpour, F. Liquid slip flow in a network of shale noncircular nanopores. Fuel 2016, 180: 580-590.

An, C., Fang, Y., Liu, S., et al. Impacts of matrix shrinkage and stress changes on permeability and gas production of organic-rich shale reservoirs. Paper SPE 186029 Presented at Reservoir Characterisation and Simulation Conference and Exhibition, Abu Dhabi, UAE, 8-10 May, 2017.

Brown, G.P., DiNardo, A., Cheng G.K., et al. The flow of gases in pipes at low pressures. J. Appl. Phys. 1946, 17(10): 802-813.

Cai, J., Lin, D., Singh, H., et al. Shale gas transport model in 3D fractal porous media with variable pore sizes. Mar. Pet. Geol. 2018, 98: 437-447.

Chai, D., Yang, G., Fan, Z., et al. Gas transport in shale matrix coupling multilayer adsorption and pore confinement effect. Chem. Eng. J. 2019, 370: 1534-1549.

Chen, S., Zhu, Y., Wang, H., et al. Shale gas reservoir characterisation: A typical case in the southern Sichuan Basin of China. Energy 2011, 36(11): 6609-6616.

Civan, F. Effective correlation of apparent gas permeability in tight porous media. Transp. Porous Media 2010, 82(2): 
375-384.

Coppens, M.O., Dammers, A.J. Effects of heterogeneity on diffusion in nanopores-from inorganic materials to protein crystals and ion channels. Fluid Phase Equilibr. 2006, 241(1-2): 308-316.

Curtis, M.E., Cardott, B.J., Sondergeld, C.H., et al. Development of organic porosity in the Woodford Shale with increasing thermal maturity. Int. J. Coal Geol. 2012, 103: 26-31.

Dong, J., Hsu, J., Wu, W., et al. Stress dependence of the permeability and porosity of sandstone and shale from TCDP Hole-A. Int. J. Rock Mech. Min. Sci. 2010, 47(7): 1141-1157.

Du, F., Nojabaei, B. A review of gas injection in shale reservoirs: Enhanced oil/gas recovery approaches and greenhouse gas control. Energies 2019, 12(12): 2355.

Freeman, C.M., Moridis, G.J., Blasingame, T.A. A numerical study of microscale flow behavior in tight gas and shale gas reservoir systems. Transp. Porous Media 2011, 90(1): 253-268.

Javadpour, F. Nanopores and apparent permeability of gas flow in mudrocks (shales and siltstone). J. Can. Pet. Technol. 2009, 48(8): 16-21.

Javadpour, F., Fisher, D., Unsworth, M. Nanoscale gas flow in shale gas sediments. J. Can. Pet. Technol. 2007, 46(10): 55-61.

Karniadakis, G., Beskok, A., Aluru, N. Microflows and Nanoflows: Fundamentals and Simulation. New York, USA, Springer Science \& Business Media, 2005.

Krishna, R., Wesselingh, J.A. The Maxwell-Stefan approach to mass transfer. Chem. Eng. Sci. 1997, 52(6): 861-911.

$\mathrm{Li}, \mathrm{C}$. , Lin, M., Ji, L., et al. Investigation of intermingled fractal model for organic-rich shale. Energy Fuels 2017, 31(9): 8896-8909.

Li, J., Chen, Z., Wu, K., et al. Effect of water saturation on gas slippage in circular and angular pores. AIChE J. 2018, 64(9): 3529-3541.

Li, X., Zhang, L. Characterization of dual-structure pore-size distribution of soil. Can. Geotech. J. 2009, 46(2): 129141.

Liu, Y., Zhu, Y. Comparison of pore characteristics in the coal and shale reservoirs of Taiyuan Formation, Qinshui Basin, China. Int. J. Coal Sci. Technol. 2016, 3(3): 330338.

Loucks, R.G., Reed, R.M., Ruppel, S.C., et al. Morphology, genesis, and distribution of nanometer-scale pores in siliceous mudstones of the Mississippian Barnett Shale. J. Sediment. Res. 2009, 79(12): 848-861.

Lu, X., Li, F., Watson, A.T. Adsorption measurements in Devonian shales. Fuel 1995, 74(4): 599-603.

Majumdar, A., Bhushan, B. Role of fractal geometry in roughness characterization and contact mechanics of surfaces. J. Tribol. 1990, 112: 205-216.

Mehmani, A., Prodanovi, M., Javadpour, F. Multiscale, multiphysics network modeling of shale matrix gas flows. Transp. Porous Media 2013, 99(2): 377-390.

Meng, M., Baldino, S., Miska, S.Z., et al. Wellbore stability in naturally fractured formations featuring dual-porosity/single-permeability and finite radial fluid discharge. J. Pet. Sci. Eng. 2019, 174: 790-803.

Mokhtari, M., Alqahtani, A.A., Tutuncu, A.N., et al. Stressdependent permeability anisotropy and wettability of shale resources. Abstracts URTeC 1555068 Presnted at Unconventional Resources Technology Conference, Denver, Colorado, 12-14 August, 2013.

Mortensen, N.A., Okkels, F., Bruus, H. Reexamination of Hagen-Poiseuille flow: Shape dependence of the hydraulic resistance in microchannels. Phys. Rev. E 2005, 71(5): 057301.

Ren, W., Li, G., Tian, S., et al. An analytical model for real gas flow in shale nanopores with non-circular cross-section. AIChE J. 2016, 62(8): 2893-2901.

Roy, S., Raju, R., Chuang, H.F., et al. Modeling gas flow through microchannels and nanopores. J. Appl. Phys. 2003, 93(8): 4870-4879.

Saurabh, S., Harpalani, S. Stress path with depletion in coalbed methane reservoirs and stress-based permeability modeling. Int. J. Coal Geol. 2018, 185: 12-22.

Sheng, G. Research on characterization of multi-scale media and flow simulation of shale gas reservoirs. Qingdao, China University of Petroleum (East China), 2019. (in Chinese)

Sheng, G., Javadpour, F., Su, Y. Effect of microscale media compressibility on apparent porosity and permeability in shale gas reservoirs. Int. J. Heat Mass Transf. 2018, 120: 56-65.

Sheng, G., Javadpour, F., Su, Y. Dynamic porosity and apparent permeability in porous organic matter of shale gas reservoirs. Fuel 2019, 251: 341-351.

Sheng, G., Zhao, H., Su, Y., et al. An analytical model to couple gas storage and transport capacity in organic matter with noncircular pores. Fuel 2020, 268: 117288.

Shi, J., Durucan, S. Drawdown induced changes in permeability of coalbeds: A new interpretation of the reservoir response to primary recovery. Transp. Porous Media 2004, 56(1): 1-16.

Singh, H., Javadpour, F. Nonempirical apparent permeability of shale. Paper URTeC 1578037 Presented at the Unconventional Resources Conference, Denver, Colorado, USA, 12-14 August, 2013.

Singh, H., Javadpour, F., Tavakkol, A.E., et al. Nonempirical apparent permeability of shale. SPE Reserv. Eval. Eng. 2014, 17(3): 414-424.

Sone, Y. Kinetic Theory and Fluid Dynamics. New York, USA, Springer Science \& Business Media, 2012.

Song, W., Wang, D., Yao, J., et al. Multiscale imagebased fractal characteristic of shale pore structure with implication to accurate prediction of gas permeability. Fuel 2019, 241: 522-532.

Wang, D., Yao, J., Chen, Z., et al. Gas-water two-phase transport properties in shale microfractures. Chinese Science Bulletin 2019, 64(31): 3232-3243. (in Chinese)

Wang, F., Jiao, L., Lian, P., et al. Apparent gas permeability, intrinsic permeability and liquid permeability of fractal porous media: Carbonate rock study with experiments and mathematical modelling. J. Pet. Sci. Eng. 2019, 173: 
1304-1315.

Wang, F., Reed, R.M. Pore networks and fluid flow in gas shales. Paper SPE 124253 Presented at Annual Technical Conference and Exhibition, New Orleans, Louisiana, 4-7 October, 2009.

Wang, G., Ju, Y. Organic shale micropore and mesopore structure characterization by ultra-low pressure $\mathrm{N}_{2}$ physisorption: Experimental procedure and interpretation model. J. Nat. Gas Sci. Eng. 2015, 27: 452-465.

Wang, X., Sheng, J. Multi-scaled pore network modeling of gas-water flow in shale formations. J. Pet. Sci. Eng. 2019, 177: 899-908.

Wheatcraft, S.W., Tyler, S.W. An explanation of scaledependent dispersivity in heterogeneous aquifers using concepts of fractal geometry. Water Resour. Res. 1988, 24(4): 566-578.

Wu, K., Chen, Z., Li, X. Real gas transport through nanopores of varying cross-section type and shape in shale gas reservoirs. Chem. Eng. J. 2015, 281: 813-825.

Wu, K., Li, X., Guo, C., et al. A unified model for gas transfer in nanopores of shale-gas reservoirs: Coupling pore diffusion and surface diffusion. SPE J. 2016, 21(5): 1583-1611.
$\mathrm{Xu}, \mathrm{J}$., Wu, K., Yang, S., et al. Real gas transport in tapered noncircular nanopores of shale rocks. AIChE J. 2017, 63(7): 3224-3242.

$\mathrm{Xu}$, S., Tang, X., Torres-Verdn, C., et al. Seismic shear wave anisotropy in cracked rocks and an application to hydraulic fracturing. Geophys. Res. Lett. 2018, 45(11): 5390-5397.

Yang, F., Ning, Z., Liu, H. Fractal characteristics of shales from a shale gas reservoir in the Sichuan Basin, China. Fuel 2014, 115: 378-384.

Yu, B., Cheng, P. A fractal permeability model for bi-dispersed porous media. Int. J. Heat Mass Transf. 2002, 45(14): 2983-2993.

Yu, B., Li, J. A geometry model for tortuosity of flow path in porous media. Chin. Phys. Lett. 2004, 21(8): 1569-1571.

Zhu, G., Kou, J., Yao, B., et al., Thermodynamically consistent modelling of two-phase flows with moving contact line and soluble surfactants. J. Fluid Mech. 2019, 879: 327359.

Zuo, H., Deng, S., Li, H. Boundary scheme for lattice Boltzmann modeling of micro-scale gas flow in organicrich pores considering surface diffusion. Chin. Phys. B 2019, 28(3): 030202. 


\section{Appendix A}

The free gas and adsorption gas permeability correction coefficients of elliptic and rectangular pore can be expressed as:

$$
\begin{gathered}
\psi_{f e m}\left(R_{\text {int }}, \varsigma_{i}\right)=\frac{\phi_{d c} l_{b}\left(R_{\text {int }}\right) n_{p}\left(R_{\text {int }}\right)\left(\pi E_{i} \varsigma_{i} R_{d c}^{2}-E_{i} \sqrt{\vartheta_{c e} \pi \varsigma_{i}} \frac{R_{d c} d_{m} p}{p_{L}+p}\right)}{\left(\frac{R_{\text {int }}}{R_{\max }}\right)^{2-2 d_{t}} \int_{R_{\min }}^{R_{\max }} l_{b}\left(R_{\text {int }}\right) n_{p}\left(R_{\text {int }}\right) \sum_{i=1}^{N} \varsigma_{i}\left(\pi E_{i} R_{d c}^{2}+4 R_{i} R_{d c}^{2}\right) d R_{\text {int }}} \\
\psi_{\text {frm }}\left(R_{\text {int }}, \varsigma_{i}\right)=\frac{4 \phi_{d c} l_{b}\left(R_{\text {int }}\right) n_{p}\left(R_{\text {int }}\right)\left[4 R_{i} \varsigma_{i} R_{d c}^{2}-4 R_{i}\left(1+\varsigma_{i}\right) \frac{R_{d c} d_{m} p}{p_{L}+p}\right]}{\left(\frac{R_{\text {int }}}{R_{\max }}\right)^{2-2 d_{t}} \int_{R_{\min }}^{R_{\max }} l_{b}\left(R_{\text {int }}\right) n_{p}\left(R_{\text {int }}\right) \sum_{i=1}^{N} \varsigma_{i}\left(\pi E_{i} R_{d c}^{2}+4 R_{i} R_{d c}^{2}\right) d R_{\text {int }}} \\
\psi_{\text {aem }}\left(R_{\text {int }}, \varsigma_{i}\right)=\frac{\phi_{d c} l_{b}\left(R_{\text {int }}\right) n_{p}\left(R_{\text {int }}\right)\left(E_{i} \sqrt{\left.\vartheta_{c e} \pi \varsigma_{i} \frac{R_{d c} d_{m} p}{p_{L}+p}\right)}\right.}{\left(\frac{R_{\text {int }}}{R_{\max }}\right)^{2-2 d_{t}}\left(\frac{d_{m}}{R_{\text {int }}}\right)^{2-d_{s}} \int_{R_{\min }}^{R_{\max }} l_{b}\left(R_{\text {int }}\right) n_{p}\left(R_{\text {int }}\right) \sum_{i=1}^{N} \varsigma_{i}\left(\pi E_{i} R_{d c}^{2}+4 R_{i} R_{d c}^{2}\right) d R_{\text {int }}} \\
\psi_{\text {arm }}\left(R_{\text {int }}, \varsigma_{i}\right)=\frac{\phi_{d c} l_{b}\left(R_{\text {int }}\right) n_{p}\left(R_{\text {int }}\right)\left[4 R_{i}\left(1+\varsigma_{i}\right) \frac{R_{d c} d_{m} p}{p_{L}+p}\right]}{\left(\frac{R_{\text {int }}}{R_{\max }}\right)^{2-2 d_{t}}\left(\frac{d_{m}}{R_{\text {int }}}\right)^{2-d_{s}} \int_{R_{\min }}^{R_{\max }} l_{b}\left(R_{\text {int }}\right) n_{p}\left(R_{\text {int }}\right) \sum_{i=1}^{N} \varsigma_{i}\left(\pi E_{i} R_{d c}^{2}+4 R_{i} R_{d c}^{2}\right) d R_{\text {int }}}
\end{gathered}
$$

